\title{
Chronic nicotine intake causes vascular dysregulation in the rat gastric mucosa
}

\author{
M Battistel, M Plebani, F Di Mario, M Jocič, I T Lippe, P Holzer
}

\begin{abstract}
Chronic cigarette smoking has adverse effects on peptic ulcer disease because the healing of ulcers is delayed and the incidence of relapses is enhanced. Short term intake of nicotine induces vascular damage in the rat gastric mucosa, but the pathophysiological mechanisms of nicotine's action in the stomach are largely unknown. In this study rats were treated with nicotine, added to their drinking water, for $\mathbf{5 0}$ days. They were then anaesthetised and their stomachs perfused with acidified acetylsalicylic acid (ASA). Chronic nicotine treatment failed to change the effects of acidified ASA to induce gastric mucosal acid back diffusion, haemorrhagic damage and bleeding. Basal blood flow in the gastric mucosa was also unchanged by chronic nicotine intake, whereas the mucosal hyperaemia evoked by ASA induced acid back diffusion was averted. The concentrations of sulfidoleukotrienes were significantly augmented in the gastric wall of nicotine treated rats. These data show that chronic nicotine intake causes dysregulation of the gastric microcirculation, an effect that is associated with biochemical changes in the stomach. This study thus substantiates the adverse effects of smoking on gastric mucosal pathophysiology. These data suggest that inappropriate regulation of gastric mucosal blood flow inhibits recovery from gastric mucosal injury in smokers.

(Gut 1993; 34: 1688-1692)
\end{abstract}

University of Graz,

Department of

Experimental and Clinical

Pharmacology, Graz,

Austria

M Battistel

M Jocič

I T Lippe

P Holzer

University of Padova, Department of

Laboratory Medicine,

Padova, Italy

M Plebani

University of Padova, Department of Internal Medicine, Padova, Italy

F Di Mario

Correspondence to:

Dr $P$ Holzer, University of Graz, Department of Experimental and Clinical Pharmacology,

Universitätsplatz 4, A-8010 Graz, Austria.

Accepted for publication 22 April 1993
Chronic cigarette smoking has adverse effects on peptic ulcer disease because the healing of ulcers is delayed and the incidence of relapses is enhanced. ${ }^{\prime}$ The aetiological causes of smoking induced gastric disease, though, are largely unknown. Nicotine, an important component of cigarette smoke, has been found to significantly influence gastric mucosal pathophysiology. When given to rats daily for 10 days, at doses simulating the daily intake of a heavy cigarette smoker, nicotine induces microscopic injury in the gastric mucosa and worsens ethanol induced damage.$^{23}$ In addition, vascular permeability is increased ${ }^{3}$ while gastric acid secretion remains unchanged. ${ }^{4}$ These findings suggest that chronic nicotine intake causes vascular damage in the rat gastric mucosa. ${ }^{3}$

Gastric mucosal blood flow is thought to play an essential part in gastric mucosal defence. ${ }^{56} \mathrm{It}$ is well known that mucosal blood flow rises when the gastric mucosal barrier is disrupted and acid diffuses back into the superficial mucosa.$^{6-9}$ This hyperaemic response is mediated by nerves ${ }^{9}$ and contributes to the prevention of acid induced mucosal injury. ${ }^{69}$ Because of the finding that chronic nicotine intake leads to vascular damage we hypothesised that nicotine may cause dysregulation of gastric mucosal blood flow thereby compromising adequate gastric mucosal defence. The aim of this study, therefore, was to investigate whether chronic nicotine intake would affect the hyperaemic response of the rat gastric mucosa to acid back diffusion.

Acetylsalicylic acid (ASA) was used in these experiments to break the gastric mucosal barrier, because this drug causes vascular damage leading to overt bleeding ${ }^{1011}$ and because vascular damage is considered to be one of the initial pathogenetic events in gastric mucosal injury. ${ }^{12}$ Local factors such as leukotrienes may have a bearing on the formation of vascular damage, although their part in gastric mucosal injury remains enigmatic. Thus, inhibition of endogenous leukotriene synthesis fails to prevent ethanol induced gastric injury, ${ }^{13+15}$ whereas exogenous sulfidoleukotrienes can aggravate the extent of damage. ${ }^{12}{ }^{16-19}$ With these considerations in mind, the gastric concentrations of sulfidoleukotrienes were determined to look for biochemical changes in the stomach of nicotine treated rats.

\section{Methods}

\section{ANIMAL PREPARATION}

Female Sprague-Dawley rats weighing 200 to $240 \mathrm{~g}$ were fasted for 20 hours but allowed free access to water. After the induction of anaesthesia with urethane $\left(1.5 \mathrm{~g} \mathrm{~kg}^{-1}\right.$ subcutaneously) the rats were fitted with a tracheal cannula to facilitate spontaneous respiration and allow hydrogen to be given. Blood pressure was recorded from a cannula in the left carotid artery by a pressure transducer and displayed on a chart recorder. The blood pressure signal was also fed into a heart ratemeter with digital readout. ${ }^{20}$ Saline $(0.9 \% \mathrm{NaCl}$, wt/wt) was continuously infused into a jugular vein at a rate of $1.5 \mathrm{ml} \mathrm{h}^{-1}$ to avoid dehydration. The stomach, exposed by a midline laparotomy, was fitted with an inflow cannula placed in the forestomach and an outflow cannula inserted through the pylorus. The stomach was perfused at a rate of $0.7-0.8 \mathrm{ml}$ $\mathrm{min}^{-1}$, and the perfusate was collected in 15 minute fractions for the determination of the hydrogen ion and haemoglobin content. The body temperature of the animals was kept at $36-37^{\circ} \mathrm{C}$ by a heating lamp. ${ }^{20}$

MEASUREMENT OF GASTRIC MUCOSAL BLOOD FLOW (MBF)

Gastric mucosal blood flow was determined by 
the hydrogen gas clearance technique. ${ }^{90}$ The clearance of hydrogen gas was measured by a needle type platinum electrode inserted from the serosa into the basal portion of the gastric mucosa and positioned at the submucosal border of the muscularis mucosae..$^{20}$ To ensure monoexponential desaturation curves, the electrode was placed in such a way that it was not in direct contact with any visible gastric artery. In those few instances in which bicomponent curves were seen, the position of the electrode was changed so that the curves became monoexponential. The construction of the electrode was identical with that described previously. ${ }^{9}$ A reference electrode was placed inside the peritoneal cavity. The current signal was amplified and recorded on a chart recorder so that the amplitude of the desaturation curves amounted to $15-20 \mathrm{~cm}$. The desaturation curves were computerised by a digitising tablet and the software SigmaScan (Jandel Scientific, Corte Madera, CA). The time needed for $50 \%$ desaturation was determined by linear regression analysis of the plot log current $v$ time, and mucosal blood flow ( $\mathrm{ml} \mathrm{min}^{-1}$ $\left(100 \mathrm{~g}^{-1}\right)$ was calculated as described previously. ${ }^{21}$

DETERMINATION OF GASTRIC ACID BACK DIFFUSION Gastric acid back diffusion was determined by the loss of $\mathrm{H}^{+}$ions from the gastric perfusate as a measure of acid back diffusion. The gastric perfusate was collected in $\mathbf{1 5}$ minute fractions, and their acid content was determined by titration of the samples to $\mathrm{pH} 7$ using $0 \cdot 1 \mathrm{~N} \mathrm{NaOH} .^{9}$ Blank samples of the perfusion media were treated in the same manner as the gastric perfusate fractions, and the loss of $\mathrm{H}^{+}$ions was determined by subtracting the acidity of the perfusate fractions from the acidity of the respective blank samples.

DETERMINATION OF GASTRIC BLEEDING

Gastric bleeding was determined by an increase in the haemoglobin concentration of the gastric perfusate. ${ }^{11}$ The content of haemoglobin was measured by spectrophotometry at $390 \mathrm{~nm}$ against rat haemoglobin as standard.

DETERMINATION OF NICOTINE

At the end of the experiments, blood $(2 \mathrm{ml})$ was withdrawn from a carotid artery, mixed with $0 \cdot 2$ $\mathrm{ml}$ of sodium citrate $(3.6 \%$, wt/wt) and frozen. The plasma content of nicotine was measured by a method similar to that described by Vereby et al. ${ }^{22}$ Briefly, blood plasma $(0.5 \mathrm{ml})$ was extracted with $3.0 \mathrm{ml}$ of $0.8 \mathrm{M}$ perchloric acid and centrifuged at $4500 \times g$ for 10 minutes. The supernatant was adjusted to $\mathrm{pH} 10$ by adding $\mathrm{NaOH}(20 \%, \mathrm{wt} / \mathrm{wt})$. After the addition of $0.4 \mathrm{ml}$ of benzene to $3.0 \mathrm{ml}$ of supernatant and recentrifugation for five minutes, samples of $10 \mu \mathrm{l}$ were injected on to a gas chromatograph/mass spectrometer using an ULTRA-1 HP column (Hewlett-Packard, Milano, Italy). The starting temperature was $70^{\circ} \mathrm{C}$ for two minutes after which the temperature rose progressively to $250^{\circ} \mathrm{C}$. Mass spectrometry was carried out by single ion detection, monitoring 84 and 162 ions for nicotine and 180 and 209 ions for ketamine as the internal standard.

DETERMINATION OF SULFIDOLEUKOTRIENES

At the end of the experiments, the stomachs were excised, opened along the greater curvature and pinned flat on a board. A biopsy specimen of about 50-100 mg full gastric wall was taken from an area of the gastric corpus that did not show gross haemorrhagic damage. The biopsy specimens were frozen, weighed, homogenised, and extracted as described previously, ${ }^{23}{ }^{24}$ the recovery of exogenous leukotrienes added to the tissue samples ranging from 89 to $95 \% .^{24}$ The content of sulfidoleukotrienes in the biopsy specimens was determined by a specific radioimmunoassay (Advanced Magnetics, Cambridge, Massachusetts, USA). The antibody used in this assay cross reacts with $\mathrm{LTC}_{4}$ (55\%), $\mathrm{LTD}_{4}(100 \%), \mathrm{LTE}_{4}(51 \%)$, and $\mathrm{LTF}_{4}$ $(67 \%)$. The identity of radioimmunologically measured leukotrienes had previously been validated by the use of high performance liquid chromatography. ${ }^{25}$

\section{EXAMINATION OF GROSS MUCOSAL INJURY}

Gross damage to the gastric glandular mucosa was determined from photographs of the stomachs pinned flat on a board. Injury was assessed by computerised planimetry. Therefore, the area that was covered by visible haemorrhagic lesions was determined by a digitising tablet and the software SigmaScan (Jandel
Figure 1: Concentration related effect of acetylsalicylic acid (ASA) in $\mathrm{HCl}(0.075 \mathrm{M})$ on gastric mucosal blood flow $(M B F)$ $H^{+}$loss from the gastric lumen, and haemoglobin $(\mathrm{Hb})$ loss (bleeding) into the gastric perfusate. HCl and ASA were given by gastric perfusion (rate: $0.75 \mathrm{ml}$ min $\left.^{-1}\right) .0$ ASA: perfusion of the stomach with $0.075 \mathrm{M}$ HCl alone. The values given show the maximal increases in $\mathrm{MBF}, \mathrm{H}^{+}$loss, and $\mathrm{Hb}$ in $M B F, H^{+}$loss, and $H$ measured during a one
loss hour period of gastric perfusion with ASA. Means (SEM), $n=5-6 .{ }^{\star} p<0.05 \mathrm{v}$ O ASA.
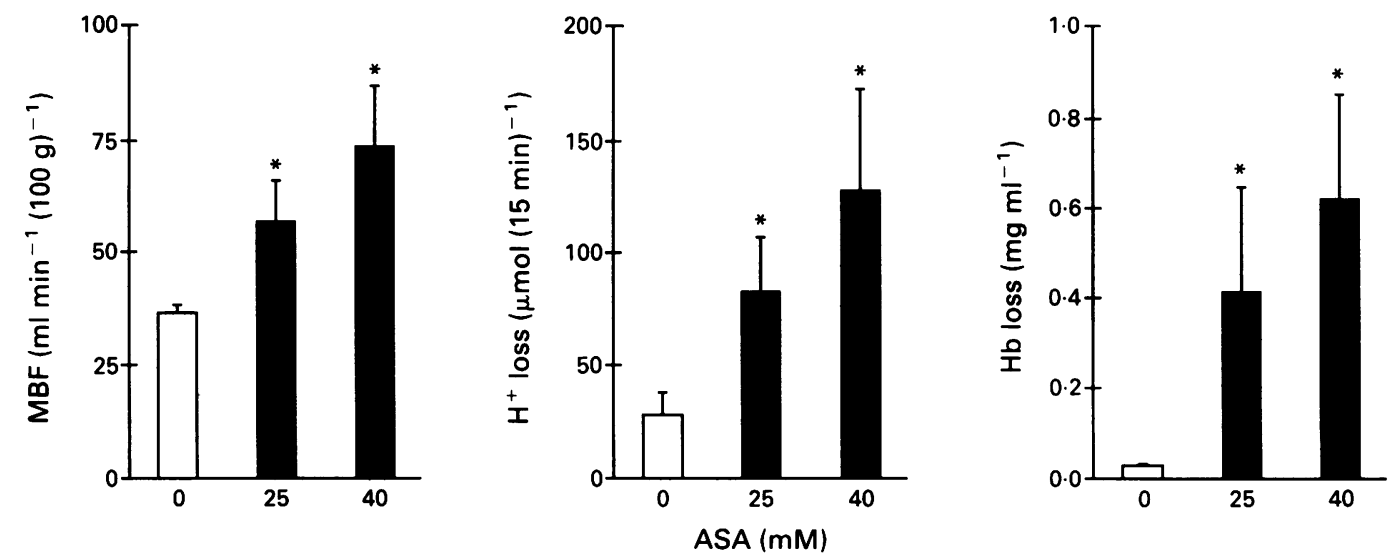
Figure 2: Time course of the effect of acetylsalicylic acid ( $A S A, 40 \mathrm{mM}$ ) in $\mathrm{HCl}$ $(0.075 M)$ on gastric mucosal blood flow (MBF), mean arterial blood pressure $(M A P)$ and heart rate (HR). $\mathrm{HCl}$ and $A S A$ were given by gastric perfusion (rate: $0.75 \mathrm{ml} \mathrm{min}^{-1}$ ). Abscissa: time from the beginning of the experiment. Means (SEM), $n=8-10$. ${ }^{\star} p<0.05,{ }^{\star \star} p<0.01 \mathrm{v}$ respective values measured immediately before administration of ASA.
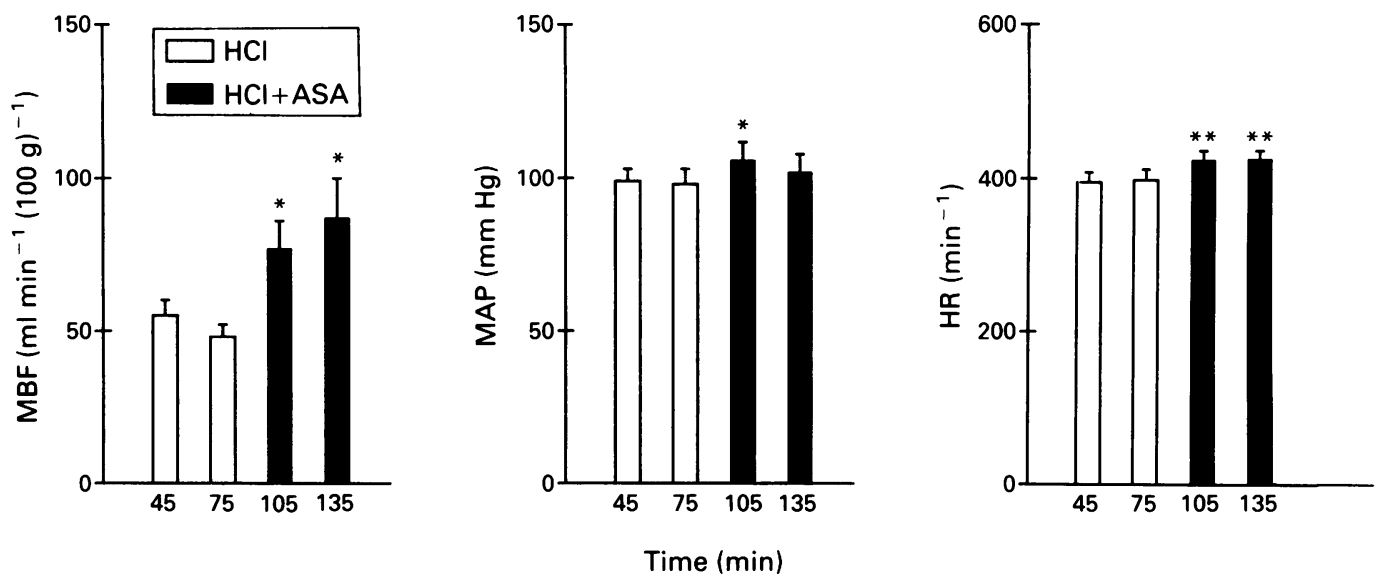

Scientific, Corte Madera, CA, USA). Gross injury was evaluated by an observer unaware of the experimental treatment. The area covered by gross injury was expressed as a percentage of the total area of the glandular mucosa of the rat stomach.

\section{EXPERIMENTAL PROTOCOL}

The experimental protocol used was equivalent to previous protocols. ${ }^{20}$ After an equilibration period of 30 minutes (experimental period 0-30 minutes), during which the stomach was perfused with saline $(0.9 \%, w t / w t)$, the perfusion medium was changed to saline containing 0.075 $\mathrm{M} \mathrm{HCl}$ and 3\% (vol/vol) TWEEN 80. Perfusion with this acid medium continued for 60 minutes (experimental period 30-90 minutes). Thereafter ASA ( 25 or $40 \mathrm{mM}$ ) was added to the acid medium, and the gastric perfusion continued for another 60 minutes (experimental period $90-150$ minutes). Mucosal blood flow was determined during the periods of 45-90, 75-90, 105-120, and 135-150 minutes. The rise in mucosal blood flow seen after ASA perfusion was expressed as mucosal blood flow increment (stimulated minus baseline values).

Two sets of experiments were carried out. In the first set, the effects of gastric perfusion with $25 \mathrm{mM}$ and $40 \mathrm{mM}$ ASA were compared with each other. In the second set of experiments rats that had drunk nicotine/tap water for 50 days were perfused with $40 \mathrm{mM}$ ASA and compared with rats that had drunk tap water alone.
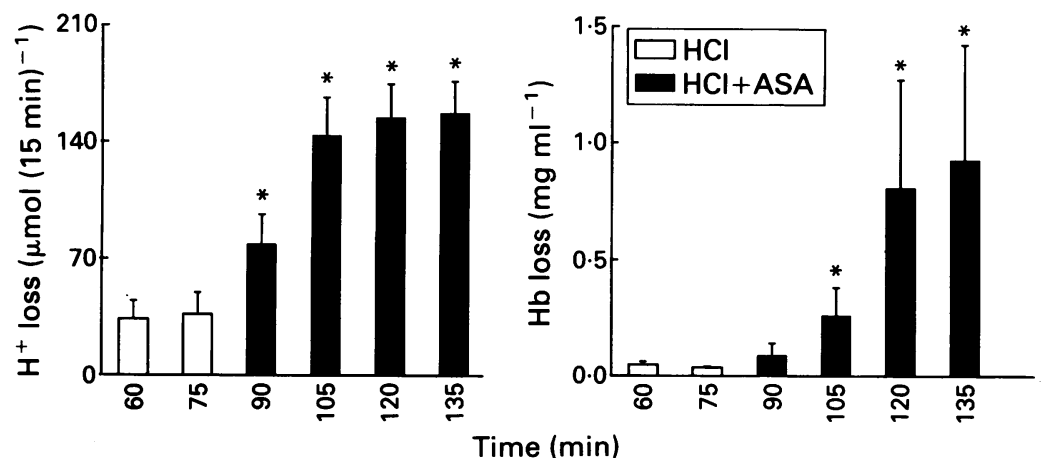

Figure 3: Time course of the effect of acetylsalicylic acid (ASA, $40 \mathrm{mM})$ in $\mathrm{HCl}(0.075 \mathrm{M})$ on $H^{+}$loss from the gastric lumen and haemoglobin $(\mathrm{Hb})$ loss (bleeding) into the gastric perfusate. $\mathrm{HCl}$ and $A S A$ were given by gastric perfusion (rate: $0.75 \mathrm{ml} \mathrm{min}^{-1}$ ). Abscissa: time from the beginning of the experiment. Means $(S E M), n=10 .{ }^{\star} p<0.05 \mathrm{v}$ respective values measured immediately before administration of ASA.

\section{NICOTINE PRETREATMENT}

Rats were allowed to drink nicotine $\left(50 \mu \mathrm{g} \mathrm{ml}^{-1}\right)$ in tap water ${ }^{3}$ for 50 days before the experiments were done.

\section{SUBSTANCES}

Nicotine was obtained from Fluka (Buchs, Switzerland) and added to tap water to give a concentration of $50 \mu \mathrm{g} \mathrm{ml}^{-1}$. ASA was purchased from Serva (Heidelberg, Germany); amounts of 2.5 or $4 \mathrm{mmol}$ were stirred in $3 \mathrm{ml}$ of TWEEN 80 for one hour, after which the appropriate amount of acid saline was added and stirring was continued until a clear solution was obtained."

\section{STATISTICS}

All data are presented as means (SEM). Statistical evaluation of the results was performed with the Student's $t$ test. Probability values $\mathrm{p}<0.05$ were regarded as significant.

\section{Results}

EXPERIMENTAL MODEL

Perfusion of the stomach with ASA increased mucosal blood flow, $\mathrm{H}^{+}$loss from the gastric perfusate, and gastric bleeding in a concentration dependent manner (Fig 1). While the increase in mucosal blood flow and $\mathrm{H}^{+}$loss became apparent within 15 minutes after gastric perfusion with $40 \mathrm{mM}$ ASA (Figs 2 and 3), it took 30 minutes until gastric bleeding was significantly enhanced (Fig 3). Apart from gastric mucosal hyperaemia, slight increases in mean arterial blood pressure and heart rate were also noted after gastric perfusion with ASA (Fig 2). ASA caused formation of haemorrhagic erosions, ${ }^{11}$ which were not assessed in these experiments.

EFFECT OF CHRONIC NICOTINE INTAKE

When rats drank tap water containing nicotine $\left(50 \mu \mathrm{g} \mathrm{m}^{-1}\right)$ for a period of 50 days, the body weight decreased slightly but significantly compared with that of the control rats (Table). Basal mucosal blood flow was the same in control and nicotine treated rats, whereas the mucosal blood flow increment induced by gastric perfusion of ASA in acid saline was significantly inhibited by 


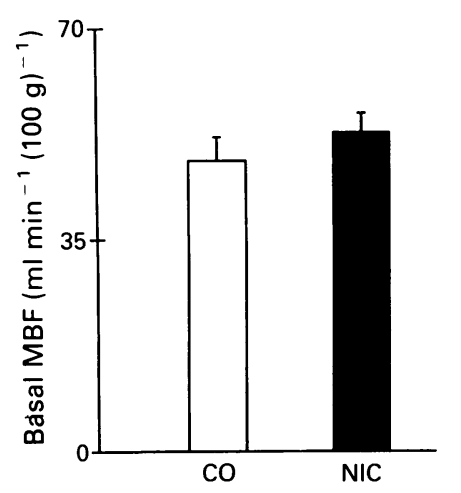

Figure 4: Gastric mucosal blood flow $(M B F)$ in control $(C O)$ rats and in rats which had drun

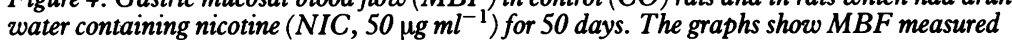
during gastric perfusion with $0.075 \mathrm{M} \mathrm{HCl}$ (basal) and the maximal increment in MBF caused by gastric perfusion with acetylsalicylic acid $(40 \mathrm{mM})$ in $\mathrm{HCl}(0.075 \mathrm{M})$. Means (SEM), $n=8-9 . \star \star p<0.01 \mathrm{v}$ respective value in the control rats.

Body weight (g), gross mucosal damage (\% of glandular mucosa), bleeding (haemoglobin content of gastric perfusate in $\left.\mu \mathrm{g} \mathrm{ml} \mathrm{l}^{-1}\right), H^{+}$loss from the gastric lumen ( $\mu \mathrm{mol}$

$\left.(15 \mathrm{~min})^{-1}\right)$, mean arterial blood pressure $(M A P, m m ~ H g)$, and heart rate $\left(H R, \mathrm{~min}^{-1}\right)$ in control rats and in rats that had drunk water containing nicotine $\left(50 \mu \mathrm{g} \mathrm{ml}^{-1}\right)$ for 50 days. The values given for bleeding, $H^{+}$loss, $M A P$, and $H R$ include both the basal values (measured during gastric perfusion with $0.075 \mathrm{M} \mathrm{HCl}$ ) and the maximal increments in the respective parameters caused by gastric perfusion with acetylsalicylic acid $(40 \mathrm{mM})$ in $\mathrm{HCl}(0.075 \mathrm{M})$

\begin{tabular}{lllll}
\hline Treatment & No & Parameter & Value & \\
\hline Control & 17 & Weight & $226(7)$ & \\
Nicotine & 18 & Weight & $211(3)^{\star}$ & \\
Control & 17 & Damage & $6 \cdot 3(1 \cdot 5)$ & \\
Nicotine & 18 & Damage & $5 \cdot 5(1 \cdot 2)$ & \\
\hline Treatment & No & Parameter & Basal & Increment \\
\hline Control & 17 & Bleeding & $29(4)$ & $+636(309)$ \\
Nicotine & 17 & Bleeding & $20(2)^{\star}$ & $+293(85)$ \\
Control & 10 & $\mathrm{H}^{+}$loss & $37(13)$ & $+133(18)$ \\
Nicotine & 10 & H loss & $33(8)$ & $+133(21)$ \\
Control & 10 & MAP & $98(5)$ & $+10(3)$ \\
Nicotine & 10 & MAP & $104(4)$ & $+9(2)$ \\
Control & 10 & HR & $398(14)$ & $+31(9)$ \\
Nicotine & 10 & HR & $405(12)$ & $+19(5)$ \\
\hline
\end{tabular}

Means $(\mathrm{SEM}) .{ }^{\star} \mathrm{p}<0.05 v$ respective control values.

chronic nicotine intake (Fig 4). Basal bleeding was reduced in nicotine treated animals, but there was no significant difference in the ASA evoked increment of bleeding between control and nicotine treated rats (Table). Basal and ASA induced gastric $\mathrm{H}^{+}$loss, mean arterial blood pressure, and heart rate did not differ significantly between control and nicotine treated animals (Table). The ASA evoked formation of
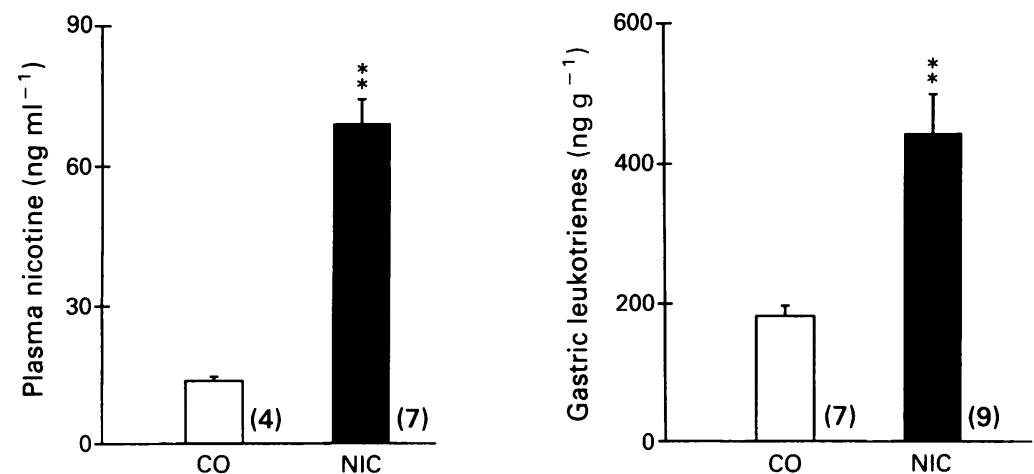

Figure 5: Concentrations of nicotine in the blood plasma (ng per $\mathrm{ml}$ ) and concentrations of sulfidoleukotrienes in the gastric corpus wall (ng per $g$ wet weight) of control $(C O)$ rats and of rats that had drunk water containing nicotine (NIC, $50 \mu \mathrm{g} \mathrm{ml}^{-1}$ ) for 50 days. The values were measured after a 60 minute perfusion of the stomach with acetylsalicylic acid $(40 \mathrm{mM})$ in $\mathrm{HCl}$ $(0.075 M)$. Means $(S E M), n$ as shown. ${ }^{\star \star} p<0.01 \mathrm{v}$ respective value in the control rats. gross mucosal damage was also unchanged by the chronic intake of nicotine (Table).

Rats, which had drunk tap water containing nicotine $\left(50 \mu \mathrm{g} \mathrm{ml}^{-1}\right)$ for a period of 50 days had significantly higher plasma concentrations of nicotine than control rats (Fig 5). The sulfidoleukotriene content of the gastric corpus wall of nicotine treated rats was significantly higher than that of control rats (Fig 5).

\section{Discussion}

The aim of this study was to examine chronic intake of nicotine over a very long time and how it affects gastric mucosal pathophysiology in the rat. Nicotine was added to the drinking water at a concentration of $50 \mu \mathrm{g} \mathrm{ml}^{-1}$, which probably simulates the daily intake of a heavy cigarette smoker. ${ }^{3}$ Nicotine was given for 50 days, a period, which in terms of life span, is roughly equivalent to 3.5 years of smoking in humans, given that the average life span of SpragueDawley rats is about 2.75 years. ${ }^{26}$ The intake of nicotine was confirmed by measuring the concentration of nicotine in the plasma of the experimental animals. The nicotine measured in the plasma of control rats represents a contamination by nicotine from various external sources. ${ }^{27}$

The effect of chronic nicotine treatment was investigated on the rat gastric mucosa challenged with acidified ASA. Unlike nicotine treatment for $\mathbf{1 0}$ days, which enhances the susceptibility of the rat gastric mucosa to ethanol injury,,$^{23}$ oral treatment of rats with nicotine for 50 days failed to change the vulnerability of the gastric mucosa by acidified ASA. After chronic nicotine administration to rats, loss of acid from the gastric lumen, formation of haemorrhagic erosions, and gastric bleeding remained unchanged, which suggests that prolonged intake of nicotine causes adaptive changes in the gastric mucosa that cancel out the adverse effects of short term treatment with nicotine. Alternatively, it may be argued that the effect of nicotine on experimental gastric injury depends on the type of the noxious agent that is used to induce damage.

ASA induced gastric damage was chosen as the experimental model of this study because ASA is able to disrupt the gastric mucosal barrier ${ }^{28}{ }^{29}$ and to significantly damage microvessels in the superficial mucosa resulting in haemorrhage and bleeding. ${ }^{111}$ Disruption of the gastric mucosal barrier by ASA, salicylate, taurocholate or ethanol is known to cause back diffusion of acid into the superficial gastric mucosa ${ }^{7929}$ as shown by an enhanced loss of $\mathrm{H}^{+}$ions from the gastric perfusate. Back diffusion of acid leads to an increase in gastric mucosal blood flow, ${ }^{6-9}$ and the rise of blood flow in the basal portion of the mucosa seen after gastric perfusion with acidified ASA is probably a result of back diffusion of acid because the degree of hyperaemia was related to the loss of acid from the gastric perfusate.

The increase in gastric mucosal blood flow caused by acid back diffusion is thought to be a protective mechanism $^{5-79}$ that may either reduce acute acid injury or aid the repair of injured mucosa, or both. The mucosal damage caused by 
acidified ASA, however, was not aggravated in nicotine treated rats, although the hyperaemic response to acid back diffusion was blunted. This finding, though, does not necessarily challenge the concept that gastric mucosal hyperaemia is an important means of defence against injury. Firstly, the adverse effects of ASA on the gastric mucosa encompass both gastric mucosal barrier disruption and vascular damage. The direct injurious effect of ASA on gastric blood vessels probably antagonises the protective action of the accompanying hyperaemia because of acid back diffusion. If so, the relation between the hyperaemic response to acidified ASA and mucosal protection may be not as clear as it is, for example, with acidified ethanol, which at low concentrations breaks the gastric mucosal barrier ${ }^{9}$ but does not disrupt gastric blood vessels. Secondly, as discussed above, longterm intake of nicotine seems to cause adaptive changes that enhance the resistance of the gastric mucosa to injury and which may, to some extent, compensate for the loss of the protective hyperaemia in response to acid back diffusion.

Chronic intake of nicotine resulted not only in functional anomalies but also in biochemical changes within the rat stomach. In this study, the sulfidoleukotriene content was used as an index of possible biochemical changes in the stomach of nicotine treated rats, because these leukotrienes constrict gastric mucosal microvessels $^{161819}$ and can predispose to vascular damage. ${ }^{1219}$ It is not possible, however, to deduce from the present findings whether the raised sulfidoleukotriene content in the stomach of nicotine treated rats has any bearing on the gastric microcirculatory changes seen in these animals.

In conclusion, this study has shown that chronic nicotine intake for 50 days causes dysregulation of the rat gastric microcirculation in that the hyperaemic response to acid back diffusion is significantly attenuated. Although acute mucosal damage induced by acidified ASA remained unchanged, it is conceivable that dysregulation of gastric mucosal blood flow in nicotine treated rats impedes recovery from injury. If this hypothesis is extrapolated to the ulcer patients who smoke, the current data would suggest that chronic intake of nicotine impairs the physiological regulation of gastric mucosal blood flow in the face of pending injury. It seems probable that this defect in gastric microcirculation is a factor relevant for the delayed ulcer healing and increased ulcer relapse rate seen in smokers.

This work was supported by the Austrian Scientific Research Council (grants P7845-MED and P9473-MED) and the Austrian National Bank (grant 4207). The authors thank Dr Ulrike HolzerPetsche for carefully reading the manuscript.

1 Walsh JH. Acid peptic disorders of the gastrointestina tract. In: Kelley WN, ed. Textbook of internal medicine. Philadelphia: Lippincott, 1989: 500-13.

2 Wong SH, Ogle CW, Cho CH. The influence of chronic or acute nicotine pretreatment on ethanol-induced gastric ulceration in the rat. $\mathcal{F}$ Pharm Pharmacol 1985; 38: 537-40.

3 Hui WM, Joana H, Chen BW, Cho CH, Luk CT, Lam SK. Nicotine induced gastric injury. A quantitative macroscopic and microscopic analysis of the protective effects of and microscopic analysis of the protectiv
sucralfate and feeding. Gut 1991; 32: 372-6.

4 Cho CH, Ogle CW, Wong SH, Lam SK. The effects of ethanol and nicotine on gastrin and somatostatin release in rats. and nicotine on gastrin and

5 Guth PH, Leung FW, Kauffman GL. Physiology of the gastric circulation. In: Schultz SG, ed. The gastrointestinal system. Handbook of physiology. Section 6, Vol I, Part 2. Bethesda: American Physiological Society, 1989: 1371-404.

6 Oates PJ. Gastric blood flow and mucosal defense. In Hollander D, Tarnawski AS, eds. Gastric cytoprotection. New York: Plenum Press, 1990: 125-65.

7 Whittle BJR. Mechanisms underlying gastric mucosal damage induced by indomethacin and bile salts, and the actions of prostaglandins. Br f Pharmacol 1977; 60: 455-60.

8 Bruggeman TM, Wood JG, Davenport HW. Local control of blood flow in the dog's stomach: vasodilatation caused by acid back-diffusion following topical application of salicylic acid back-diffusion following topical ap

9 Holzer P, Livingston EH, Guth PH. Sensory neurons signal for an increase in rat gastric mucosal blood flow in the face of pending acid injury. Gastroenterology 1991; 101: 416-23.

10 Kitahora T, Guth PH. Effect of aspirin plus hydrochloric acid on the gastric mucosal microcirculation. Gastroenterology 1987; 93: 810-7.

11 Holzer P, Pabst MA, Lippe ITh. Intragastric capsaicin protects against aspirin-induced lesion formation and bleeding in the rat gastric mucosa. Gastroenterology 1989; 96 : $1425-33$.

12 Pihan G, Rogers C, Szabo S. Vascular injury in acute gastric mucosal damage. Mediatory role of leukotrienes. $\mathrm{Dig} \mathrm{Dis} S \mathrm{Ci}$ 1988; 33: 625-32.

13 Wallace JL, Beck PL, Morris GP. Is there a role for leukotrienes as mediators of ethanol-induced gastric leukotrienes as mediators of ethanol-induced
mucosal damage? Am $\mathcal{F}$ Physiol 1988; 254: G117-23.

14 Boughton-Smith NK, Whittle BJR. Failure of the inhibition of rat gastric mucosal 5-lipoxygenase by novel acetohydroxamic acids to prevent ethanol-induced damage. $\mathrm{Br} \mathcal{F}$ Pharmacol 1988; 95: 155-62.

15 Peskar BM, Trautmann M, Respondek M, Müller KM. Role of prostaglandins and leukotrienes in healing of ethanolinduced gastric mucosal damage in rats. Gastroenterology 1991; 100: A619.

16 Whittle BJR, Oren-Wolman N, Guth PH. Gastric vasoconstrictor actions of leukotriene $\mathrm{C}_{4}, \mathrm{PGE}_{2 \alpha}$, and thromboxane mimetic U-46619 on rat submucosal microcirculation in vivo. Am 7 Physiol 1985; 248: G580-6.

17 Peskar BM, Hoppe U, Lange K, Peskar BA. Effects of nonsteroidal anti-inflammatory drugs on rat gastric mucosal steroidal anti-inflammatory drugs on rat gastric mucosal induced injury. Brf Pharmacol 1988; 93: 937-43.

18 Yonei Y, Guth PH. Lipoxygenase metabolites in the rat gastric microvascular responses to intragastric ethanol. Gastroenterology 1989; 97: 304-12.

19 Wallace JL, McKnight GW, Keenan CM, Byles NIA MacNaughton WK. Effects of leukotrienes on susceptibility of the rat stomach to damage and investigation of the mechanism of action. Gastroenterology 1990; 98: 1178-86.

20 Holzer P, Lippe ITh. Gastric mucosal hyperemia due to acid backdiffusion depends on splanchnic nerve activity. $A m \mathcal{F}$ Physiol 1992; 262: G505-9.

21 Leung FW, Guth PH, Scremin OA, Golanska EM, Kauffman GL. Regional gastric mucosal blood flow measurements by hydrogen gas clearance in the anesthetized rat and rabbit. hydrogen gas clearance in the antroestogy 1984; 87: 28-36.
Gastroentol

22 Vereby G, De Pace A, Mule SJ, Kanzeler O, Jaffe JH. Rapid, quantitative GLC method for the simultaneous determination of nicotine and cotinine. $\mathcal{F}$ Anal Toxicol 1982; 6: 294-6.

23 Plebani M, Di Mario F, Dal Santo PL, Faggian D, Germana $\mathrm{B}$, Vianello F, et al. Measurement of pepsinogen group I in endoscopic gastroduodenal biopsies. Clin Chem 1990; 36 : $682-4$.

24 Mattoli S, Masiero M, Calabrò F, Mezzetti M, Plebani M, Allegra L. Eicosanoid release from human bronchia epithelial cells upon exposure to toluene diisocyanate in vitro. F Cell Physiol 1990; 142: 379-85.

25 Zocca E, Fabbri LM, Boschetto P, Plebani M, Masiero M, Milani GF, et al. Leukotriene $B_{4}$ and late asthmatic reactions induced by toluene diisocyanate. $\mathcal{J}$ Appl Physiol 1990; 68: 1576-80.

26 Baker $\mathrm{HJ}$, Lindsey JR, Weisbroth SH. The laboratory rat. Biology and diseases. Vol 1 . New York: Academic Press, 1979.

27 Feyerabend C, Russell MAH. Assay of nicotine in biological materials: sources of contamination and their elimination. f Pharm Pharmacol 1980; 32: 178-81

28 Rowe PH, Starlinger MJ, Kasdon E, Hollands MJ, Silen W Parenteral aspirin and sodium salicylate are equally injurious to the rat gastric mucosa. Gastroenterology 1987; 93: 863-71.

29 Kauffman G. Aspirin-induced gastric mucosal injury: lesson learned from animal models. Gastroenterology 1989; 96 : 606-14. 